\title{
FARMACOGENÉTICA DO TRATAMENTO DE DIABETE MELITO
}

\author{
PHARMACOGENETICS OF THE DIABETES MELLITUS TREATMENT
}

Rosario Dominguez Crespo Hirata, Mario Hiroyuki Hirata

\begin{abstract}
Docentes. Departamento de Análises Clínicas e Toxicológicas. Faculdade de Ciências Farmacêuticas - USP. Correspondência: Rosario D.C. Hirata. Av. Prof. Lineu Prestes, 580 B. 17. CEP 05508-900 São Paulo - SP Tel.: (11) 3091-3660. FAX: (11) 3813-2197. E-mail: rosariohirata@usp.br
\end{abstract}

Hirata RDC, Hirata MH. Farmacogenética do tratamento de diabete melito. Medicina (Ribeirão Preto) 2006; 39 (4): 554-61.

RESUMO: Diabete melito é uma doença metabólica caracterizada pela deficiência ou resistência de insulina que leva a complicações micro e macrovasculares graves. Fatores genéticos e ambientais contribuem para a etiologia complexa das principais formas de diabete. Os avanços recentes na genética molecular contribuíram para a identificação de vários locos e genes que são relacionados aos subtipos de diabete clinicamente reconhecidos. Este conhecimento tornou-se muito importante para o reconhecimento de pacientes diabéticos com resposta diferencial a fármacos hipoglicemiantes específicos. Neste estudo serão revisados os aspectos moleculares das formas monogênicas e poligênicas da diabete assim como a abordagem farmacogenética dos agentes terapêuticos mais comuns.

Descritores: Diabete Melito Tipo 1. Diabete Melito Tipo 2. Farmacogenética. Insulina. Hipoglicemiantes Orais.

\begin{tabular}{ll} 
& \multicolumn{1}{c}{ ABREVIATURAS } \\
DM1 & $\begin{array}{l}\text { diabete melito tipo 1 } \\
\text { hemoglobina glicada }\end{array}$ \\
HLA & $\begin{array}{l}\text { antígenos leucocitários humanos } \\
\text { enzima conversora de angiotensina }(A C E)\end{array}$ \\
ACE & apolipoproteina E \\
GCK & glicoquinase \\
IFG1 & fator de crescimento insulina-símile 1 \\
INS & $\begin{array}{l}\text { insulina (INS) } \\
\text { receptor de proliferador de peroxissoma ati- }\end{array}$ \\
PPARG & $\begin{array}{l}\text { vado } \gamma \\
\text { diabete melito tipo 2 }\end{array}$ \\
DM2 & $\begin{array}{l}\text { Maturity Onset Diabetes of the Young, } \\
\text { MODY }\end{array}$ \\
HNF-4 $\alpha$ & $\begin{array}{l}\text { fator de transcrição nuclear de hepatócito } \\
4 \alpha\end{array}$
\end{tabular}

HNF-1 $\alpha$ fator de transcrição nuclear de hepatócito $1 \alpha$

IPF1 fator 1 promotor de insulina

HNF-1 $\beta$ fator de transcrição nuclear de hepatócito $1 \beta$

IB1 fator 1 cerebral de ilhota 1

GNB3 subunidade $\beta 3$ da proteína $G$

IRS1 substrato 1 do receptor de insulina quinase

GLUT4 transportador de glicose 4

GSY1 glicogênio sintase muscular

SUR1 receptor 1 de sulfuniluréia

ABC ATP binding cassette

IKATP canal de potássio dependente de ATP

TZDs tiazolidenedionas

TNDM diabete melito neonatal transitória

PNDM diabete melito neonatal permanente 


\section{1- INTRODUÇÃO}

A diabete melito é uma doença caracterizada por deficiência ou resistência a insulina que leva a complicações micro e macrovasculares graves. A diabete tem alta prevalência no mundo e, atualmente, acomete mais de 150 milhões de pessoas. A diabete é classificada em tipo 1 e tipo 2, de acordo com a etiologia que é multifatorial. Fatores genéticos têm um papel importante em várias formas de diabete, mas a herança é complexa e interage com fatores ambientais. Embora a maioria dos casos de diabete sejam distúrbios poligênicos, várias formas monogênicas foram identificadas. O tratamento é instituído de acordo com o tipo de diabete e compreende a administração de insulina ou hipoglicemiantes orais. A resposta a esses agentes terapêuticos também é influenciada por fatores genéticos e ambientais. Nesta revisão são descritos os determinantes genéticos da resposta farmacológica na diabete.

\section{2- TIPOS DE DIABETE MELITO}

A diabete melito tipo 1 (DM1) é uma doença heterogênea caracterizada por uma insuficiência absoluta de insulina causada por destruição autoimune das células- $\beta$ do pâncreas em indivíduos geneticamente predispostos a doença ${ }^{1}$. A DM1 representa 5 a $10 \%$ dos casos de diabete e é mais comumente diagnosticada em crianças e adolescentes ( $75 \%$ dos casos). A DM1 se apresenta nas formas clássica, silenciosa e cetoacidose diabética. O tratamento da DM1 visa normalizar a glicemia e a hemoglobina glicada (HbA1c) e prevenir a cetoacidose diabética e a hipoglicemia, procurando manter o desenvolvimento orgânico normal e controlar e prevenir as complicações micro e macrovasculares da doença.

A DM1 tem etiologia multifatorial com fortes componentes genéticos e ambientais. Os componentes ambientais compreendem a exposição a agentes virais (ex, enterovirus, rubéola e outros), toxinas ambientais (ex., nitrosaminas) ou alimentos (ex., exposição precoce a proteínas do leite de vaca, cereais, glúten ou soja) $)^{2,3}$.

A DM1 não tem um padrão mendeliano de herança. Os principais determinantes genéticos de susceptibilidade à DM1 são os genes do complexo dos antígenos leucocitários humanos (HLA) que participam na apresentação de antígenos e representam 40$60 \%$ da susceptibilidade genética. Os locos predomi- nantes são HLA DR (DR3/DR4) e DQA (DQA/ $\mathrm{DQB})$ que contribuem para $50 \%$ de risco de $\mathrm{DM} 1^{2,3}$. Muitos outros locos e genes candidatos localizados na maioria dos cromossomos têm sido associados com a susceptibilidade ou a resistência a DM1, tais como genes da enzima conversora de angiotensina $(A C E)$, apolipoproteina $\mathrm{E}(A P O E)$, glicoquinase $(G C K)$, fator de crescimento insulina-símile 1 (IGF1), insulina (INS), receptor de proliferador de peroxissoma ativado $\gamma$ $(P P A R G)$, entre outros ${ }^{1}$.

A diabete tipo 2 (DM2) é uma síndrome heterogênea que resulta de deficiência na secreção de insulina pelas células- $\beta$ do pâncreas e hiperglicemia por aumento das gliconeogenese hepática devido a resistência à insulina no músculo esquelético ${ }^{4}$. A DM2 é uma doença multifatorial com complexa interação entre fatores genéticos e ambientais que influenciam uma série de fenótipos intermediários (ex., massa de células- $\beta$, secreção de insulina, ação de insulina, distribuição de gordura, obesidade) ${ }^{5}$.

Os determinantes genéticos da DM2 monogênica são conhecidos e estão associados com o inicio precoce da diabete (Maturity Onset Diabetes of the Young, MODY) ${ }^{6,7}$. A diabete mitocondrial é outra forma de DM2 causada por mutações no DNA mitocondrial que levam a diminuição da fosforilação oxidativa $^{8}$. As formas poligênicas da DM2 são mais difíceis de determinar porque resultam de combinações de alterações em múltiplos genes ${ }^{4}$.

MODY é um grupo de seis diferentes formas de DM2 monogênica de herança autossômica dominante que geralmente se desenvolve na infância, adolescência ou juventude e ocorre por deficiência de secreção de insulina pelas células- $\beta^{5}$. As características de dois grupos de MODY são apresentadas no Quadro I. A maioria dos casos de MODY resulta de alterações em seis genes: glicoquinase $(G C K /$ MODY2), fator de transcrição nuclear de hepatócito $4 \alpha$ (HNF4/MODY1), HNF-1 $\alpha$ (HNF 1/MODY3), fator 1 promotor de insulina (IPF1/MODY4), HNF-1 $\beta$ (HNF1/MODY5) e NeuroD1/ $\beta 2^{9 / 15}$. Outros genes podem estar associados com MODY (MODY-X), pois em 14 a 45\% de casos não se observa co-segregação com os marcadores MODY conhecidos.

Vários genes têm sido associados com a forma poligênica e tardia da DM2, incluindo os genes MODY. Outros fatores de transcrição, tais como o fator 1 cerebral de ilhota 1 (IBI), PPARG, HNF1, HNF4 e subunidade $\beta 3$ da proteína $\mathrm{G}$ (GPB3), entre outros genes, também foram associados com DM2 $2^{5,16}$. Estudos 
Quadro I. Caracteristicas clínicas de dois subgrupos de MODY

\begin{tabular}{lll}
\hline \multirow{2}{*}{ Características Clínicas } & MODY2 & Outros MODY \\
\cline { 2 - 3 } & Glicoquinase & HNF1 $\alpha$ (MODY3), HNF1 $\beta$ (MODY5), HNF4 $\alpha$ (MODY1) \\
\hline Início & Nascimento & Adolescência/adulto jovem \\
Hiperglicemia & Estável & Progressiva \\
Tratamento & Dieta & $1 / 3$ dieta, $1 / 3$ hipoglicemiantes orais, $1 / 3$ insulina \\
Complicações & Raras & Freqüentes \\
\hline
\end{tabular}

MODY: Maturity Onset Diabetes of the Young.

de nosso grupo de pesquisa e outros mostraram forte associação entre os polimorfismos Pro12Ala e C161T do gene PPARG e a resistência à insulina em pacientes com DM2 ${ }^{17,18,19}$. As alterações nos genes dos fatores de transcrição (PPARG, HFN1, HNF4 e outros) contribuem para o risco de DM2 devido a falha na regulação de genes do metabolismo glicídico e lipídico, desenvolvimento e diferenciação anormal de células$\beta$, e falha na regulação de apoptose de células- $\beta^{4}$.

Mutações na região promotora do gene INS foram associadas com redução da transcrição e hipoinsulinemia relativa ou absoluta ${ }^{4}$. Alterações na região hipervariável do gene INS (INS-VNTR) foram associados com menor sensibilidade à insulina ${ }^{20}$. A DM2 também foi associada com variantes de genes do receptor da insulina, substrato 1 do receptor de insulina quinase (IRS1), transportador de glicose 4 (GLUT4), glicogênio sintase muscular (GSY1), GNB3 e outros ${ }^{4,16}$.
O canal de potássio dependente de ATP (IKATP) das células- $\beta$ tem um papel central na secreção de insulina induzida pela glicose. IKATP é composto de duas subunidades: uma que forma o poro do canal iônico (Kir6.2) e uma subunidade regulatória, receptor 1 de sulfuniluréia (SUR1), que pertence a superfamília de transportadores ATP binding cassette $(\mathrm{ABC})^{21 / 24}$. Mutações nos genes que codificam as subunidades Kir6.2 (KCNJ11) e SUR1 ( $A B C C 8)$ foram associadas com a diabete neonatal ${ }^{25 / 30}$. No Quadro II, são apresentadas algumas das mutações do gene $K C N J 11$ que estão associadas com diabete neonatal.

\section{3- TRATAMENTO DA DIABETE MELITO}

O controle glicêmico é fundamental para a prevenção de complicações micro e macrovasculares da diabete e pode ser realizado pela administração de hipo-

Quadro II. Tipos de diabete melito associados com variantes do gene Kir6.2 (KCNJ11)

\begin{tabular}{llcc}
\hline Tipos & Variantes & $\begin{array}{c}\text { Gravidade } \\
\text { clínica }\end{array}$ & $\begin{array}{c}\text { Gravidade } \\
\text { funcional }\end{array}$ \\
\hline Diabete melito tipo 2 & E23K & + & + \\
TNDM & G53S, G53R, I182V & + & + \\
PNDM isolada & R201H, Y330C, Y330S, R50Q, R50P & ++ & ++ \\
DEND intermediaria & V59M, R201C & +++ & +++ \\
Sindrome DEND & Q52R, V59G, C166Y, C166F, 296V & ++++ & ++++ \\
\hline
\end{tabular}

PNDM: diabete melito neonatal permanente.

Síndrome DEND: síndrome de retardo no desenvolvimento, eplepsia e diabete neonatal.

TNDM: diabete melito neonatal transitória 
glicemiantes orais ou insulina. A HbA1c é o marcador principal para avaliação do controle glicêmico e deve ser mantida abaixo de 7\%, segundo a Associação Americana de Diabete ${ }^{31}$.

Os hipoglicemiantes orais são fármacos utilizados no tratamento da DM2 e são classificados como biguanidas, sulfoniluréias, meglitinidas, e tiazolidenedionas e acarbose ${ }^{32}$.

As biguanidas, como a metimorfina, atuam nos tecidos hepático, muscular e adiposo por diferentes mecanismos, melhorando a função das células- $\beta$ do pâncreas e a resistência insulínica ${ }^{31,32}$. A metimorfina diminui a produção hepática e aumenta a captação muscular de glicose pelo acoplamento a AMP quinase, enzima de sinalização que desencadeia uma série de processos metabólicos celulares, incluindo a oxidação de ácidos graxos e a geração de ATP. A metimorfina reduz a insulina circulante sem causar hipoglicemia e diminui as complicações macrovasculares da diabete em $30 \%$. A metimorfina é geralmente bem tolerada, mas os pacientes podem apresentar desconforto gastrintestinal e náuseas ocasionalmente.

As sulfaniluréias e as meglitinidas são agentes estimulantes da secreção de insulina pelas células- $\beta$ do pâncreas ${ }^{31,32}$. As sulfaniluréias inibem a atividade do canal de potássio dependente de ATP (IKATP) que resulta na despolarização da membrana celular e leva ao influxo de cálcio e liberação de insulina pelas células. O aumento da insulina circulante suprime a produção hepática de glicose e promove a captação de glicose pelo músculo esquelético e tecido adiposo.

As tiazolidenedionas (TZDs) são ligantes do PPAR $\gamma$, fator de transcrição expresso em células-alvo da ação da insulina, principalmente nos adipócitos ${ }^{4,31,32}$. Quando ativado, o PPAR $\gamma$ modula a expressão de genes envolvidos no metabolismo lipídico, na diferenciação do tecido adiposo e na ação da insulina. O principal efeito metabólico é o aumento da sensibilidade a insulina nos tecidos periféricos, principalmente o músculo esquelético e, em altas doses, as TZDs também reduzem a produção hepática de glicose. As TZDs tem efeitos adversos potenciais como ganho de peso e retenção de liquido que resultam da expansão do tecido adiposo subcutâneo e aumento da retenção de sódio e água livre.

A acarbose é um inibidor de $\alpha$-glicosidase que retarda a absorção de glicose no intestino delgado, reduzindo a magnitude da hiperglicemia no período pósprandial $^{31,32}$. Os principais efeitos adversos que podem limitar o seu uso são entumescimento abdominal, flatulência e diarréia em mais de $30 \%$ dos pacientes.
A insulina é utilizada individualmente ou em combinação com hipoglicemiantes orais, quando o controle glicêmico mais agressivo é necessário, como é o caso da DM1 ${ }^{31}$. Embora a hiperinsulinemia endógena esta associada com complicações cardiovasculares, a insulinoterapia é segura e não desencadeia eventos cardíacos adversos. Também terapia intensiva com insulina em certas condições agudas, como o infarto agudo do miocárdio, pode reduzir a morbidade e a mortalidade.

\section{4- FATORES FARMACOGENÉTICOS DA DIABETE MELITO}

O diagnóstico molecular das formas monogênicas da DM2 é importante porque tem efeito significativo no tratamento. Os pacientes com MODY2 não têm necessidade de tratamento farmacológico enquanto que os portadores de MODY3 são muito sensíveis a baixas doses de sulfoniluréias ${ }^{23}$. Além disso, os pacientes com diabete neonatal causada por mutações no gene KCNJ11, apesar de serem dependentes de insulina, podem interromper a insulinoterapia e são bem controlados com altas doses de sulfoniluréia ${ }^{33}$. O desafio para os endocrinologistas é usar as habilidades clínicas para detectar pacientes com DM2 monogênicas, cujos cuidados poderão ser significativamente auxiliados pelas alterações no tratamento após o teste genético molecular ${ }^{23}$.

\section{1- Farmacogenética da diabete do adulto jovem (MODY)}

Pacientes com MODY2 tem mutações no gene $G C K$, sensor da glicose intracelular, e apresentam hiperglicemia de jejum moderada ao nascimento que se deteriora muito pouco com a idade (Quadro III). O tratamento é geralmente dietético sem risco de complicações microvasculares ${ }^{34}$. Não há diferenças em HbA1c entre pacientes tratados ou não tratados e a interrupção do tratamento não resulta na deterioração do controle da glicemia ${ }^{33}$. Os testes genéticos para MODY em indivíduos diabéticos não são freqüentemente realizados devido ao custo e sua indisponibilidade na rotina laboratorial. Entretanto, ao avaliar o impacto do teste genético para MODY2 nos custos de controle e tratamento da diabete em uma criança de 5 anos, verificou-se que há redução em $25 \%$ do custo utilizado no tratamento com insulina. Portanto há benefícios financeiros e para a saúde no diagnostico de MODY2 que deve ser recomendado em pacientes específicos. 
Recentemente foi desenvolvida uma nova droga hipoglicemiante que atua como ativador da glicoquinase ${ }^{35,36}$. Essa terapia tem melhorado a resposta hipoblicemiante e o peso ao nascimento em portadores de variante comum localizada na região promotora do gene $G C K^{37}$.

Pacientes com MODY3 (HNF1 $\alpha$ ) são normoglicêmicos ao nascimento e posteriormente desenvolvem hiperglicemia progressiva que resulta em forma de diabete grave (Quadro III). A maioria dos pacientes requer tratamento farmacológico. Pacientes com MODY3 (HNF1 $\alpha$ ) podem ser extremamente sensíveis aos efeitos hipoglicemiantes de sulfoniluréias nas doses padrões ${ }^{38}$. Em um estudo cruzado randomizado recente, pacientes com MODY3 tiveram resposta quatro vezes maior a sulfoniluréia glilazida que os pacientes com DM2 ${ }^{39}$. A resposta farmacogenética foi atribuída a maior secreção de insulina em resposta a sulfoniluréia e não ao metabolismo alterado da droga. $\mathrm{O}$ controle glicêmico pode deteriorar acentuadamente quando a sulfoniluréia substituída por metimorfina. Portanto, apesar da metimorfina ser o tratamento farmacológico principal para a DM2, para os pacientes com MODY3 o tratamento com sulfoniluréia é mais adequado e tem impacto significante na qualidade de vida.

A resposta terapêutica da MODY5 (HNF-1 $\beta$ ) é diferente da MODY3 (HNF1 $\alpha$ ). Isto é surpreendente considerando a alta homologia entre os dois fatores de transcrição e o fato que eles tem um sitio de ligação comum, mas provavelmente reflete diferenças no tempo e nos locais de expressão ${ }^{33}$. Os pacientes portadores de MODY5 são mais freqüentemente tratados com insulina e não tem sensibilidade a sulfoniluréia como os pacientes com MODY3.

\section{2- Farmacogenética da diabete neonatal}

Há recentes avanços na genética e tratamento da diabete neonatal que é usualmente diagnosticada nos primeiros três meses de vida. Há duas formas de diabete neonatal: diabete melito neonatal transitória (TNDM) que se resolve, usualmente dentro de três meses; e a diabete melito neonatal permanente (PNDM) que pode requer a vida toda insulinoterapia. A maioria dos pacientes com TNDM tem uma anormalidade de imprinting do gene ZAC e HYMAI no cromossomo $6 \mathrm{q}^{33}$.

Mutações no gene $K C N J 11$ que codifica a subunidade do IKATP Kir6. 2 das células- $\beta$ foram detectadas em 31-64\% dos pacientes com PNDM $^{40}$. Essas mutações prejudicam o fechamento do IKATP na presença de ATP, o que resulta em influxo de potássio aumentado e mantém a membrana da célula- $\beta$ em estado hiperpolarizado, bloqueando a secreção de insulina.

Pacientes com mutações no gene KCNJI1 podem ser tratados com sulfoniluréias em substituição à injeção de insulina (Quadro III). As sulfoniluréias têm maior capacidade de ligar-se ao SUR1 e fechar o IKATP por uma via independente de $\mathrm{ATP}^{41}$. Portadores de variantes do gene $K C N J 1$ tiveram o tratamento insulínico descontinuado utilizando glibenclamida ou outras sulfoniluréias, alguns dos quais apresentaram melhor controle glicêmico ${ }^{42,43}$. Estes foram alguns dos primeiros casos em que pacientes dependentes de insulina puderam ser tratados eficientemente com sulfoniluréias.

\section{3- Farmacogenética da DM2 poligênica}

Foi observado que 5-7\% de pacientes diabéticos tratados com sulfoniluréia tem seu tratamento

Quadro III. Etiologia, genes envolvidos e tratamento de diferentes formas de diabete

\begin{tabular}{lll}
\hline Etiologia & Gene afetado & Tratamento \\
\hline Diabete melito tipo 1 & HLA DR/DQA & Insulina \\
Diabete melito tipo 2 & PPARG & Metiformina \\
MODY2 & Glicoquinase & Nenhum \\
MODY3 & Fator nuclear hepático 1 $\alpha$ & Sulfoniluréia - doses baixas \\
Diabete neonatal & Subunidade do canal de potássio Kir6.2 & Sulfoniluréia - doses altas \\
\hline
\end{tabular}

MODY: Maturity Onset Diabetes of the Young. 
substituído por insulina por não responderem a sulfoniluréia ${ }^{44}$. Este fenômeno clínico foi denominado falha secundária a sulfoniluréia e tem sido associado com aumento no peso corporal, regime dietético inadequado, diagnostico na idade jovem, deterioração na sensibilidade a insulina ou presença de anticorpos anti-ilhota e anti-GAD ${ }^{4}$. A deterioração do controle glicêmico na DM2 foi associada com a diminuição da função das células- $\beta$ independente da dieta e da monoterapia intensiva. Foi observado que o IRS-1 tem um papel importante na deterioração progressiva da função das células- $\beta$ que pode levar a falha na terapia com sulfoniluréias. Estudos recentes demonstraram que variantes dos genes IRSI e KCNJII estão associadas com risco aumentado de falha secundária ao tratamento com sulfoniluréias, representando exemplos potenciais de farmacogenética da DM2 $2^{44,45}$.

Uma fração significativa de pacientes tratados com TZD (30-40\%) não responde ao tratamento com melhora da sensibilidade a insulina ${ }^{46}$. Estudo recente pesquisou mutações no gene $P P A R G$, em mulheres com diabete gestacional prévia, e verificaram que oito va- riantes foram associadas com a resposta a $\mathrm{TZD}^{47}$. Os autores do estudo concluíram que alterações no gene $P P A R G$ podem ser responsáveis pela resposta a TZD em mulheres em risco de DM2.

\section{5- CONCLUSÕES E PERSPECTIVAS}

A identificação de genes associados com o risco de diabete tem contribuído para a compreensão dos mecanismos moleculares que mantém a homeostase da glicose e dos defeitos moleculares que levam a hiperglicemia crônica. Novos esquemas terapêuticos têm sido propostos para melhorar o controle glicêmico e a qualidade de vida dos pacientes diabéticos, reduzindo significativamente a morbidade e mortalidade associada com a diabete e suas complicações.

A abordagem farmacogenética da diabete possibilita estudar o papel de determinantes genéticos no sucesso da terapia com insulina e hipoglicemiantes orais. Além de contribuir para a predição da resposta terapêutica, os estudos farmacogenéticos são fundamentais para a descoberta de novos alvos terapêuticos.

Hirata RDC, Hirata MH. Pharmacogenetics of the diabetes mellitus treatment. Medicina (Ribeirão Preto) 2006;39 (4): 554-61.

ABSTRACT: Diabetes mellitus is a metabolic disease involving the deficiency or resistance to insulin that triggers to severe micro and macrovascular complications. Both genetic and environmental factors contribute to the complex etiology of main forms of diabetes. The recent advances in molecular genetics contributed to the identification of several loci and candidate genes that are related to the clinically recognized diabetes subtypes. This knowledge has become more important for recognition of diabetic patients with differential response to specific hypoglycemic drugs. In this study, the molecular aspects of the monogenic and polygenic forms of diabetes as well as the pharmacogenetics approach of the drugs commonly used in treatment are reviewed.

Keywords: Diabetes Mellitus, Type I. Diabetes Mellitus, Type II. Pharmacogenetics. Insulin. Hypoglycemic, Oral.

\section{REFERÊNCIAS}

1 - Atkinson MA. ADA Outstanding Sc c Achievement Lecture 2004. Thirty years of investigating the autoimmune basis for type 1 diabetes: why can't we prevent or reverse this disease? : Diabetes 2005; 54: 1253-63.

2 - Haller MJ, Atkinson MA, Schatz D. Type 1 diabetes mellitus: etiology, presentation, and management. Pediatr Clin North Am 2005; 52: 1553-78.
3 - Daneman D. Type 1 diabetes. Lancet. 2006; 367: 847-58.

4 - Petersen KF, Shulman GI. Etiology of insulin resistance. Am J Med 2006; 119 (Suppl 1): S10-6.

5 - Froguel P, Velho G. Genetic determinants of type 2 diabetes. Recent Prog Horm Res 2001; 56: 91-105.

6 - Fajans SS, Bell GI, Polonsky KS. Molecular mechanisms and clinical pathophysiology of maturity-onset diabetes of the young. N Engl J Med 2001; 345: 971-80. 
7 - Stride A, Hattersley AT. Different genes, different diabetes: lessons from maturity-onset diabetes of the young. Ann Med 2002; 34: 207-16.

8 - Maassen JA, T Hart LM, Van Essen E, Heine RJ, Nijpels G, Jahangir Tafrechi RS, Raap AK, Janssen GM, Lemkes HH. Mitochondrial diabetes: molecular mechanisms and clinical presentation. Diabetes 2004; 53 (Suppl 1): S103-9.

9 - Winter WE. Molecular and biochemical analysis of the MODY syndromes. Pediatr Diabetes 2000; 1: 88-117.

10 - Pearson ER, Velho G, Clark P, Stride A, Shepherd M, Frayling TM, TM, Bulman MP, Ellard S, Froguel P, Hattersley AT. betacell genes and diabetes: quantitative and qualitative differences in the pathophysiology of hepatic nuclear factor-1alpha and glucokinase mutations. Diabetes 2001; 50 (Suppl 1): S101-7.

11 - Ryffel GU. Mutations in the human genes encoding the transcription factors of the hepatocyte nuclear factor (HNF)1 and HNF4 families: functional and pathological consequences. J Mol Endocrinol 2001; 27: 11-29.

12 - Gloyn AL. Glucokinase (GCK) mutations in hyper- and hypoglycemia: maturity-onset diabetes of the young, permanent neonatal diabetes, and hyperinsulinemia of infancy. Hum Mutat 2003; 22: 353-62.

13 - Giuffrida FM, Reis AF. Genetic and clinical characteristics of maturity-onset diabetes of the young. Diabetes Obes Metab 2005; 7: 318-26.

14 - Pearson ER, Pruhova S, Tack CJ, Johansen A, Castleden HA, Lumb PJ, TM, Bulman MP, Ellard S, Froguel P, Hattersley AT. Molecular genetics and phenotypic characteristics of MODY caused by hepatocyte nuclear factor 4alpha mutations in a large European collection. Diabetologia 2005; 48: 878-85.

15 - Stride A, , Clark P, Shakespeare L, Salzmann M, Shepherd M, Hattersley AT. Beta-cell dysfunction, insulin sensitivity, and glycosuria precede diabetes in hepatocyte nuclear factor1alpha mutation carriers. Diabetes Care 2005; 28: 1751-6.

16 - Fernandez-Real JM, Penarroja G, Richart C, Castro A, Vendrell J, Broch M, Lopez-Bermejo A, Ricart W.. G protein beta3 gene variant, vascular function, and insulin sensitivity in type 2 diabetes. Hypertension 2003; 41: 124-9.

17 - Altshuler D, Hirschhorn JN, Klannemark M, Lindgren CM, Vohl MC, Nemesh J, Lane CR, Schaffner SF, Bolk S, Brewer C, Tuomi T, Gaudet D, Hudson TJ, Daly M, Groop L, Lander ES.. The common PPARgamma Pro12Ala polymorphism is associated with decreased risk of type 2 diabetes. Nat Genet 2000; 26: 76-80.

18 - Tavares V, Hirata RD, Rodrigues AC, Monte O, Salles JE, Scalissi N, Speranza AC, Hirata MH. Association between Pro12Ala polymorphism of the PPAR-gamma2 gene and insulin sensitivity in Brazilian patients with type-2 diabetes mellitus. Diabetes Obes Metab 2005; 7: 605-11.

19 - Tavares V, Hirata RD, Rodrigues AC, Monte O, Salles JE, Scallissi N, . Effect of the peroxisome proliferator-activated receptor-gamma $\mathrm{C} 161 \mathrm{~T}$ polymorphism on lipid profile in Brazilian patients with Type 2 diabetes mellitus. J Endocrinol Invest 2005; 28: 129-36.

20 - Ong KK, Dunger DB. Birth weight, infant growth and insulin resistance. Eur J Endocrinol 2004; 151 (Suppl 3) : U131-9.
21 - Florez JC, Burtt N, de Bakker PI, Almgren P, Tuomi T, Holmkvist J, Gaudet D, Hudson TJ, Schaffner SF, Daly MJ, Hirschhorn JN, Groop L, Altshuler D. Haplotype structure and genotype-phenotype correlations of the sulfonylurea receptor and the islet ATP-sensitive potassium channel gene region. Diabetes 2004; 53: 1360-8.

22 - Del Guerra S, Lupi R, Marselli L, Masini M, Bugliani M, Sbrana S, Torri S, Pollera M, Boggi U, Mosca F, Del Prato S, Marchetti P. Functional and molecular defects of pancreatic islets in human type 2 diabetes. Diabetes 2005; 54: 727-35.

23 - Hattersley AT. Molecular genetics goes to the diabetes clinic. Clin Med 2005; 5: 476-81.

24 - Koster JC, Permutt MA, Nichols CG. Diabetes and insulin secretion: the ATP-sensitive $\mathrm{K}+$ channel (K ATP) connection. Diabetes 2005; 54: 3065-72.

25 - Gloyn AL, Pearson ER, Antcliff JF, Proks P, Bruining GJ, Slingerland AS, Howard N, Srinivasan S, Silva JM, Molnes J, Edghill EL, Frayling TM, Temple IK, Mackay D, Shield JP, Sumnik Z, van Rhijn A, Wales JK, , Gorman S, , Ellard S, Njolstad PR, Ashcroft FM, Hattersley AT. Activating mutations in the gene encoding the ATP-sensitive potassium-channel subunit Kir6.2 and permanent neonatal diabetes. N Engl J Med 2004; 350: 1838-49.

26 - Proks P, Antcliff JF, Lippiat J, Gloyn AL, Hattersley AT, Ashcroft FM. Molecular basis of Kir6.2 mutations associated with neonatal diabetes or neonatal diabetes plus neurological features. Proc Natl Acad Sci U S A 2004; 101: 17539-44.

27 - Hattersley AT, Ashcroft FM. Activating mutations in Kir6.2 and neonatal diabetes: new clinical syndromes, new scientific insights, and new therapy. Diabetes 2005; 54: 2503-13.

28 - Flanagan SE, Edghill EL, Gloyn AL, Ellard S, Hattersley AT. Mutations in KCNJ11, which encodes Kir6.2, are a common cause of diabetes diagnosed in the first 6 months of life, with the phenotype determined by genotype. Diabetologia 2006;49:1190-7.

29 - Gloyn AL, Siddiqui J, Ellard S. Mutations in the genes encoding the pancreatic beta-cell KATP channel subunits Kir6.2 (KCNJ11) and SUR1 (ABCC8) in diabetes mellitus and hyperinsulinism. Hum Mutat 2006; 27: 220-31.

30 - Proks P, Arnold AL, Bruining J, Girard C, Flanagan SE, Larkin B, Colclough K, Hattersley AT, Ashcroft FM, Ellard S. A heterozygous activating mutation in the sulphonylurea receptor SUR1 (ABCC8) causes neonatal diabetes. Hum Mol Genet 2006; 15:1793-800.

31 - Rosenberg DE, Jabbour SA, Goldstein BJ. Insulin resistance, diabetes and cardiovascular risk: approaches to treatment. Diabetes Obes Metab 2005; 7: 642-53.

32 - Liberopoulos EN, Tsouli S, Mikhailidis DP, Elisaf MS. Preventing type 2 diabetes in high risk patients: an overview of lifestyle and pharmacological measures. Curr Drug Targets 2006; 7: 211-28.

33 - Hattersley AT, Pearson ER. Mini Review: Pharmacogenetics and beyond: the interaction of therapeutic response, betacell physiology and genetics in diabetes. Endocrinology 2006; 147: 2657-63.

34 - Schnyder S, Mullis PE, Ellard S, Hattersley AT, Fluck CE. Genetic testing for glucokinase mutations in clinically selected patients with MODY: a worthwhile investment. Swiss Med Wkly 2005; 135: 352-6. 
35 - Grimsby J, Sarabu R, Corbett WL, Haynes NE, Bizzarro FT, Coffey JW, Guertin KR, Hilliard DW, Kester RF, Mahaney PE, Marcus L, Qi L, Spence CL, Tengi J, Magnuson MA, Chu CA, Dvorozniak MT, Matschinsky FM, Grippo JF. Allosteric activators of glucokinase: potential role in diabetes therapy. Science 2003; 301: 370-3

36 - Matschinsky FM, Magnuson MA, Zelent D, Jetton TL, Doliba N, Han Y, Tabú R, Grimsby J. The network of glucokinaseexpressing cells in glucose homeostasis and the potential of glucokinase activators for diabetes therapy. Diabetes 2006; 55: 1-12.

37 - Weedon MN, Frayling TM, Shields B, Knight B, Turner T, Metcalf BS, Voss L, Wilkin TJ, McCarthy A, Ben-Shlomo Y, Davey Smith G, Ring S, Jones R, Golding J, Byberg L, Mann V, Axelsson T, Syvanen AC, Leon D, Hattersley AT. Genetic regulation of birth weight and fasting glucose by a common polymorphism in the islet cell promoter of the glucokinase gene. Diabetes 2005; 54: 576-81.

38 - Pearson ER, Liddell WG, Shepherd M, Corrall RJ, Hattersley AT. Sensitivity to sulphonylureas in patients with hepatocyte nuclear factor-1alpha gene mutations: evidence for pharmacogenetics in diabetes. Diabet Med 2000; 17: 543-5.

39 - Pearson ER, Starkey BJ, Powell RJ, Gribble FM, Clark PM, Hattersley AT. Genetic cause of hyperglycaemia and response to treatment in diabetes. Lancet 2003; 362 (9392): $1275-81$

40 - Sagen JV, Raeder H, Hathout E, Shehadeh N, Gudmundsson K, Baevre H, Abuelo D, Phornphutkul C, Molnes J, Bell GI, Gloyn AL, Hattersley AT, Molven A, Sovik O, Njolstad PR. Permanent neonatal diabetes due to mutations in KCNJ11 encoding Kir6.2: patient characteristics and initial response to sulfonylurea therapy. Diabetes 2004; 53: 2713-8.

41 - Koster JC, Remedi MS, Dao C, Nichols CG. ATP and sulfonylurea sensitivity of mutant ATP-sensitive $\mathrm{K}+$ channels in neonatal diabetes: implications for pharmacogenomic therapy. Diabetes 2005; 54: 2645-54.
42 - Zung A, Glaser B, Nimri R, Zadik Z. Glibenclamide treatment in permanent neonatal diabetes mellitus due to an activating mutation in Kir6.2. J Clin Endocrinol Metab 2004; 89: 5504-7.

43 - Slingerland AS, Hattersley AT. Mutations in the Kir6.2 subunit of the KATP channel and permanent neonatal diabetes: new insights and new treatment. Ann Med 2005; 37: 186-95.

44 - Sesti G, Marini MA, Cardellini M, Sciacqua A, Frontoni S, Andreozzi F, Irace C, Lauro D, Gnasso A, Federici M, Perticone F, Lauro R. The Arg972 variant in insulin receptor substrate-1 is associated with an increased risk of secondary failure to sulfonylurea in patients with type 2 diabetes. Diabetes Care 2004; 27: 1394-8.

45 - Sesti G, Laratta E, Cardellini M, Andreozzi F, Del Guerra S, Irace C, Gnasso A, Grupillo M, Lauro R, Hribal ML, Perticone $F$, Marchetti $P$. The E23K variant of KCNJ11 encoding the pancreatic \{beta\}-cell KATP channel subunits Kir6.2 is associated with an increased risk of secondary failure to sulfonylurea in patients with type 2 diabetes. J Clin Endocrinol Metab 2006; 91: 2334-9.

46 - Buchanan TA, Xiang AH, Peters RK, Kjos SL, Marroquin A, Goico J, Ochoa C, Tan S, Berkowitz K, Hodis HN, Azen SP. Preservation of pancreatic beta-cell function and prevention of type 2 diabetes by pharmacological treatment of insulin resistance in high-risk hispanic women. Diabetes 2002; 51: $2796-803$

47 - Wolford JK, Yeatts KA, Dhanjal SK, Black MH, Xiang AH, Buchanan TA, Watanabe RM. Sequence variation in PPARG may underlie differential response to troglitazone. Diabetes 2005; 54: 3319-25 